\title{
Sensor Fusion for Semantic Segmentation of Urban Scenes
}

\author{
Richard Zhang ${ }^{1}$ Stefan A. Candra ${ }^{2}$ Kai Vetter ${ }^{3}$ Avideh Zakhor ${ }^{1}$
}

\begin{abstract}
Semantic understanding of environments is an important problem in robotics in general and intelligent autonomous systems in particular. In this paper, we propose a semantic segmentation algorithm which effectively fuses information from images and 3D point clouds. The proposed method incorporates information from multiple scales in an intuitive and effective manner. A late-fusion architecture is proposed to maximally leverage the training data in each modality. Finally, a pairwise Conditional Random Field (CRF) is used as a postprocessing step to enforce spatial consistency in the structured prediction. The proposed algorithm is evaluated on the publicly available KITTI dataset [1] [2], augmented with additional pixel and point-wise semantic labels for building, sky, road, vegetation, sidewalk, car, pedestrian, cyclist, sign/pole, and fence regions. $A$ per-pixel accuracy of $89.3 \%$ and average class accuracy of $65.4 \%$ is achieved, well above current state-of-the-art [3].
\end{abstract}

\section{INTRODUCTION}

Semantic segmentation involves labeling every pixel in an image, or point in a point cloud, with its corresponding semantic tag. A semantic understanding of the environment facilitates robotics tasks such as navigation, localization, and autonomous driving.

Most approaches to semantic segmentation on images follow a CRF framework. Exceptions include the methods proposed by Farabet et al. [4] and Girshick et al. [5], who use expressive features learned from convolutional neural nets. Tighe et al. [6] [7], Singh et al. [8], and Yang et al. [9] use a image retrieval system followed by non-parametric classification to produce per-superpixel classifications, with special considerations are taken for objects [7], semantic context [8], and rare classes [9]. The per-superpixel classifications are then placed in a CRF framework to balance local beliefs while enforcing spatial consistency, where unary potentials are per-superpixel classifications, and pairwise connections are made between neighboring superpixels.

Point cloud semantic segmentation methods typically follow the same bottom-up pipeline [10] [11] [12] [13] [14]. They start with an oversegmentation, followed by regional feature extraction, classification, and a CRF.

A challenge in the bottom-up approach is the difficulty in integrating and enforcing top-down information and structure. Kohli et al. [15] formulate Robust $P^{N}$ clique potentials. The technique was used by Ladicky et al. [16] to incorporate

\footnotetext{
$\begin{array}{cccc}{ }^{1} \text { Department of } & \text { Electrical } & \text { Engineering and } & \text { Com- } \\ \text { puter } & \text { Science, } & \text { University of California, } & \text { Berkeley. }\end{array}$ \{rich.zhang, avz\}@eecs.berkeley.edu

${ }^{2}$ Department of Computer Science, University of California, Berkeley candrastefandberkeley .edu

${ }^{3}$ Department of Nuclear Engineering, University of California, Berkeley and the Nuclear Science Division, Lawrence Berkeley National Lab kvetteralbl.gov
}

sliding window object detections, and extended into a multilevel hierarchical manner by Ladicky et al. [17]. Using clique potentials comes at a cost of run-time, and solving for optimal parameters remains an open problem.

A cascade of classifiers in a hierarchical scheme was proposed by Munoz et al. [18] and applied in the image domain, and later extended by Xiong et al. [19] on point clouds. A hierarchical segmentation is first performed, and an initial classifier is trained on segments in the coarsest segmentation. A classifier is trained on the subsequent segmentation level, with the outputs from the previous stage incorporated as input features, and this process is repeated. Information can be passed downwards or upwards multiple times through the hierarchy. The method is extended to integrate information across modalities by Munoz et al. [20], where classifications are passed to the subsequent level of the segmentation hierarchy in both modalities.

Several previous studies in semantic segmentation of urban 3D data have been recently performed [21] [22] [3]. Sengupta et al. [21] and He et al. [22] first perform semantic segmentation in the image modality. The image labeling results are projected into 3D using point clouds extracted from stereo vision, and results are aggregated in 3D using a voting scheme. Both methods disregard point cloud features. Cadena and Košecká [3] propose a pipeline to solve the semantic segmentation problem using a CRF framework, with an emphasis on fast run-time. The method first oversegments the image and extracts simple low-dimensional features. An early fusion architecture is used, where separate feature sets are extracted based on sensor coverage of the segment. A CRF framework is used to enforce spatial consistency. The method is designed to run quickly on a coarse label set, but struggles on fine object classes such as pedestrian and fence.

In this paper, we propose an algorithm for semantic parsing of the environment using information from multiple sensing modalities, namely images and 3D point clouds. In contrast to previous work [3] [20] [17], we explicitly take into account information from multiple scales up front by first performing segmentation on multiple scales and performing inference on the fine segmentation scale. Feature vectors of the low-level segments are augmented with features computed on the corresponding segments from higherlevel segmentations. This is different from previous work [3], which uses localized features computed on a single scale only. Our approach precludes the need to train an expanding number of classifiers [20] or rely on complex graphical model machinery [17], but allows us to effectively integrate information from multiple scales.

Unlike [3], which uses early fusion, we fuse information 


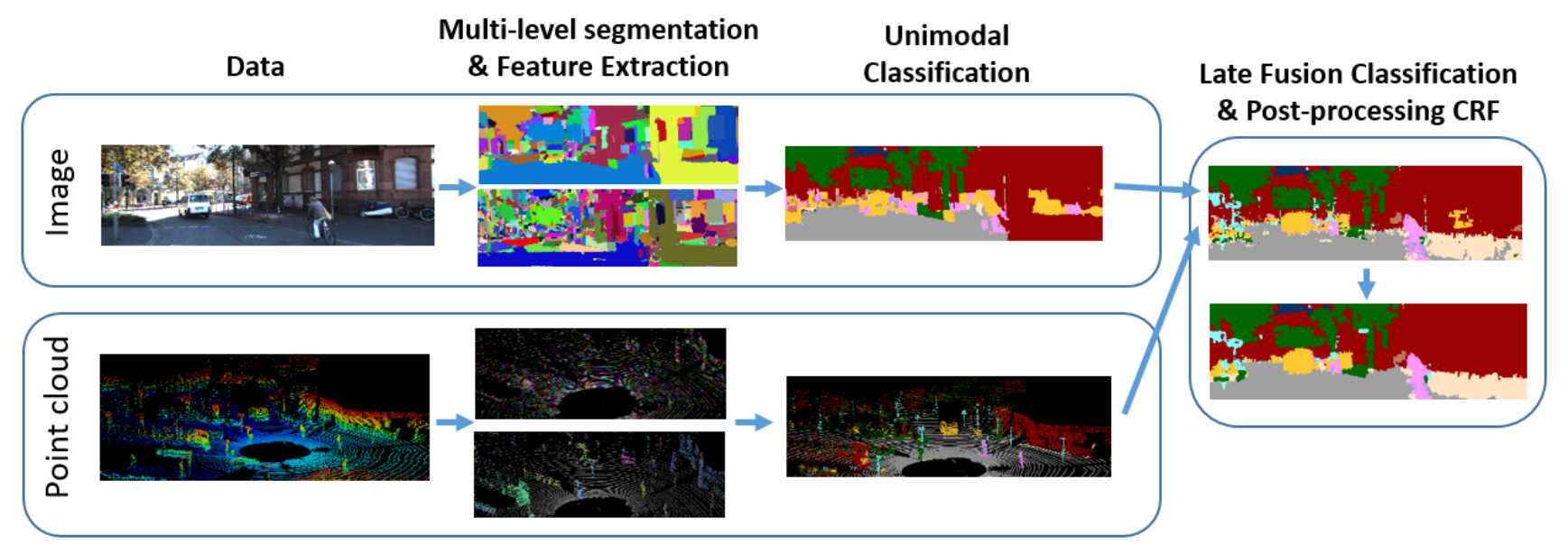

Fig. 1: Top-level pipeline.

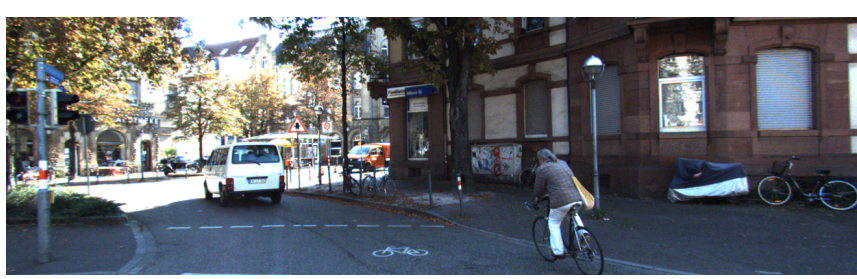

(a)

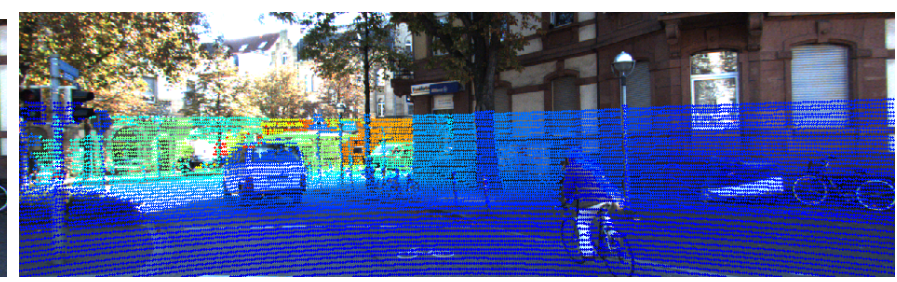

(b)

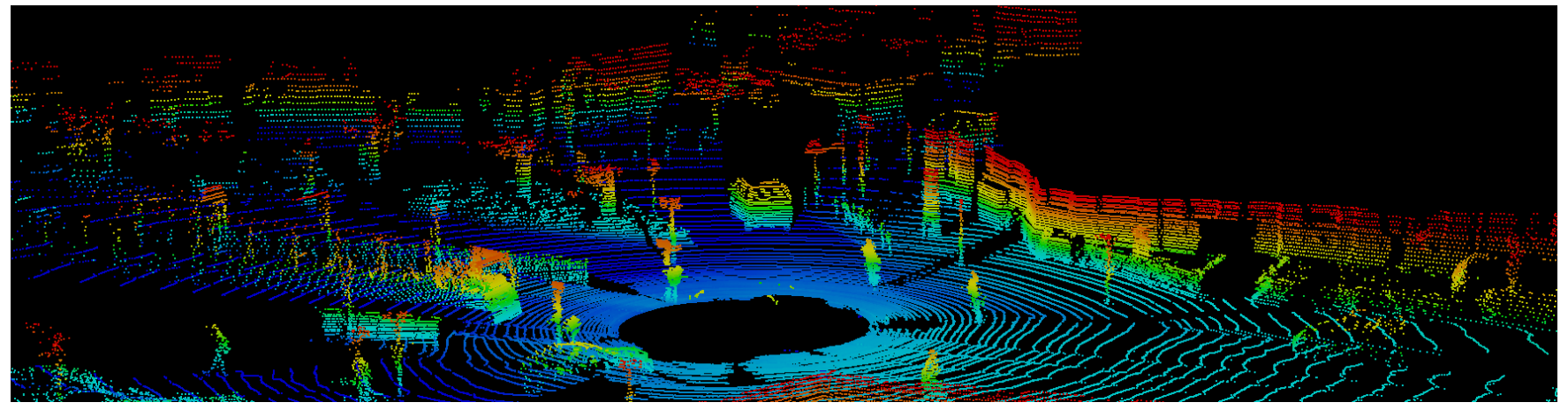

(c)

Fig. 2: Example acquisition; (a) image; (b) point cloud projected onto image, colored by depth; (c) point cloud, colored by height.

using a late fusion architecture. Specifically, a fusion classifier is used on regions with overlapping sensor coverage is trained on the soft classifications of the unimodal classifiers. Late fusion enables us to effectively leverage the data from the entire training set, rather than the subset with overlapping sensor coverage. Finally, we use a pairwise CRF as a post-processing step to enforce spatial consistency. We test on the publicly available KITTI platform [1] [2], which contains images and registered point clouds, and demonstrate improvement over the current state of the art [3].

The outline of the paper is as follows. Section II provides an overview of the proposed algorithm. Section III details experimental results. We conclude and discuss future directions of study in Section IV.

\section{ALGORITHM DESCRIPTION}

A top-level pipeline of our algorithm is illustrated in Figure 1. The input to our algorithm is individual acquisitions of an image and its corresponding 3D point cloud, taken from the KITTI platform, as shown in Figures 2(a) and 2(c), respectively. The camera and lidar sensors are calibrated with respect to each other; Figure 2(b) shows the example point cloud projected onto an image.

As seen in Figure 1, our algorithm is comprised of the following steps:

- Multi-level segmentation: Segmentation is first performed in each modality at two levels: a low or finer level and a high or coarser level. Segments on the lowlevel segmentation are used to perform inference.

- Feature Extraction: Each segment from the low level is associated to an overlapping segment from the higher- 


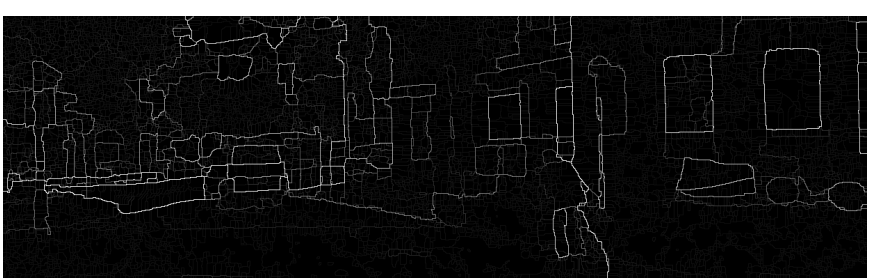

(a)

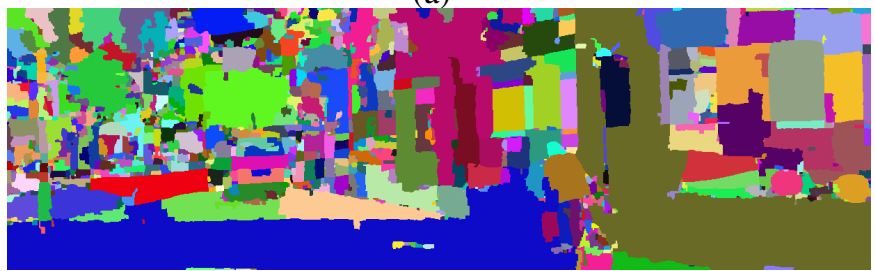

(c)

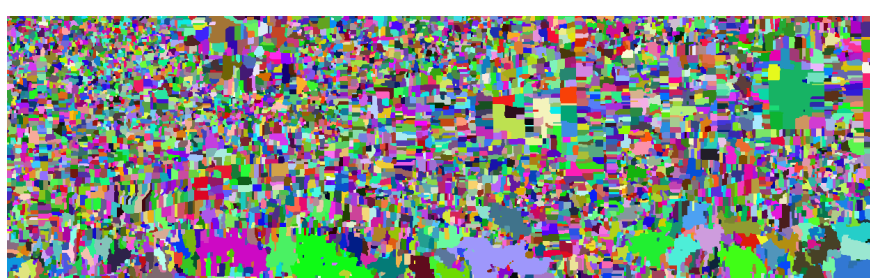

(b)

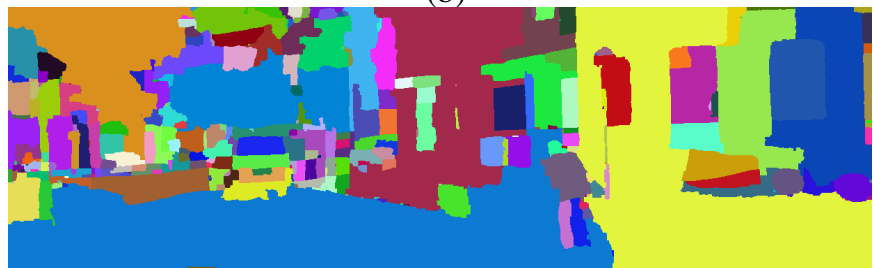

(d)

Fig. 3: Example image segmentation; (a) UCM [23]; Superpixels generated using thresholds t = (b) 0.06 ; (c) 0.1 ; (d) 0.2 .

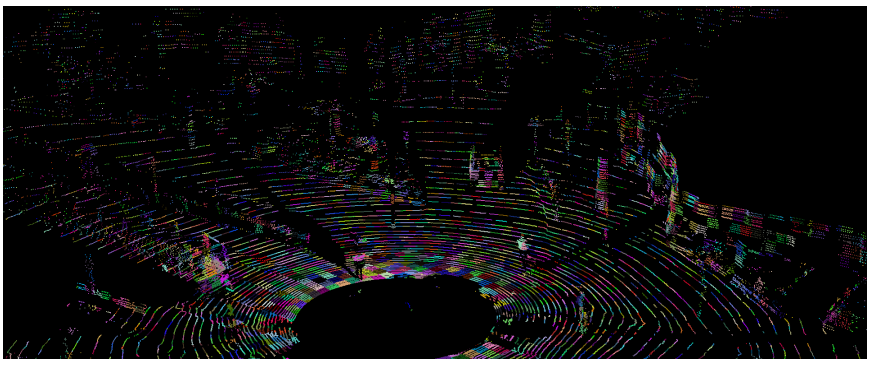

(a)

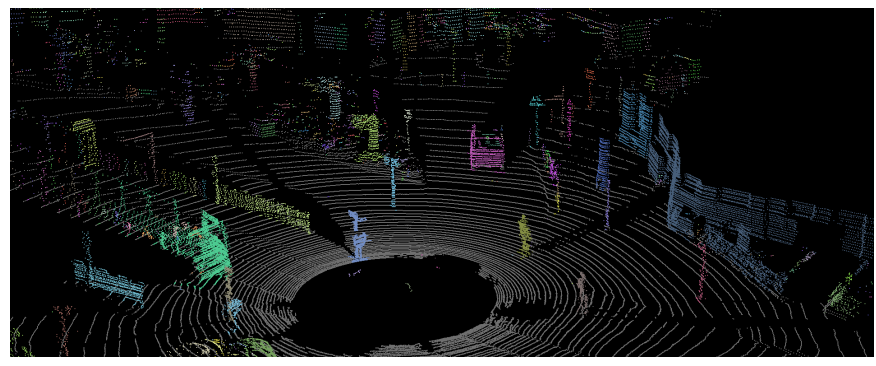

(b)

Fig. 4: Example point cloud segmentation; (a) Fine segmentation via VCCS [24]; (b) Coarse segmentation via connected component segmentation.

level. Features are extracted from the low-level segments, and concatenated with the features from their associated high-level segments.

- Classification and Fusion: Segments are first classified using unimodal image or point cloud-only classifiers. Segments which are within the overlapping coverage of both sensing modalities are then reclassified by fusing the outputs from the unimodal classifiers.

- Post-processing CRF: A pairwise CRF is used as postprocessing step to enforce spatial consistency.

We describe each of the above steps in more detail below.

\section{A. Segmentation}

In order to effectively capture information at multiple scales, two levels of segmentation are performed. In the image modality, an ultrametric contour map (UCM) is obtained by using the Multiscale Combinatorial Grouping algorithm [23]. The UCM is the equivalent representation of a hierarchical segmentation, and can be converted to a segmentation by thresholding at level $t$, with lower values of $t$ producing a finer set of superpixels. For our experiments, we empirically found values of $t_{1}=0.10$ and $t_{2}=0.20$ to work well. Example low and high-level segmentations are shown in Figures 3(c) and 3(d), respectively.

In the point cloud modality, we use the Voxel Cloud Connectivity Segmentation (VCCS) algorithm [24] to produce fine-level segments. The algorithm uses region growing to produce uniformly sized supervoxels while respecting object boundaries. We choose to produce supervoxels of dimension approximately $0.5 \mathrm{~m}$ for our experiments. We generate highlevel segmentations from a connected component segmentation, similar to [25]. Points are binned into a $0.1 \times 0.1 \mathrm{~m} 2 \mathrm{D}$ grid in the $x$ and $y$ dimensions. The difference in height of the points within each cell $\Delta z$ is computed. Cells with $\Delta z$ above and below $0.1 \mathrm{~m}$ are labeled occupied and unoccupied, respectively. Connected components are extracted on the occupied cells. RANSAC plane fit is run on points in unoccupied cells to obtain a ground plane estimate. Points above the ground estimate which lie within occupied cells of the same connected component belong to the same segment. An example point cloud segmentation is shown in Figure 4. As seen in Figure 4(b), connected component segments can vary in size and encompass cars, people, or entire building façades on the order of $10 \mathrm{~m}$.

\section{B. Feature Extraction}

We extract features for each segment at each scale. Specifically, the features at the fine level contain bottom-up information, such as color and texture, but do not contain as much top-down shape information. Since we have segmented at multiple scales, each low-level segment can be associated to high-level segment. The feature vector for each low-level 
segment is then augmented with features extracted from the associated segment on the high-level segmentation.

We compute features for each superpixel in an image, similar to those in [6], as summarized in Table I(a). In particular, we compute size, shape, position, color features, and a high dimensional SIFT [26] Bag-of-words (BoW) feature. To incorporate contextual information, we also dilate the bounding box of the superpixel by 10 pixels on each side and compute color and SIFT BoW features on this region, excluding the superpixel mask. The same features are computed on the high-level segmentation, with some exclusions. Specifically, the contextual features are not computed on the larger segment, since the segments are already large. The high-dimensional descriptors are more suited for low-level bottom-up classification and are also not computed.

Table I(b) shows the features we choose to extract on the point cloud. In particular, we compute size, shape, position, and orientation features on both the segmentation levels. PCA analysis on a segment determines eigenvalues $\lambda_{i}$, where $\lambda_{1} \geq \lambda_{2} \geq \lambda_{3}$, and corresponding eigenvectors $\mathbf{v}_{\mathbf{i}}=\left(v_{i x} v_{i y} v_{i z}\right)^{T}$. We define $\Lambda=\sum_{i=1}^{3} \lambda_{i}$. Similar to the image modality, the high-dimensional BoW descriptor, which in this case are Spin Images [27], is suited to bottomup classification and is thus only computed on the low-level segmentation.

TABLE I: Features extracted.

\begin{tabular}{|c|c|c|c|c|}
\multicolumn{5}{c|}{ (a) Image Features. } \\
\hline Type & Name & Dim & Low & High \\
\hline \multirow{4}{*}{ Size/Shape } & Area & 1 & $\checkmark$ & $\checkmark$ \\
\hline & Equivalent Diameter & 1 & $\checkmark$ & $\checkmark$ \\
\hline & Major/minor axes & 2 & $\checkmark$ & $\checkmark$ \\
\hline & Orientation & 1 & $\checkmark$ & $\checkmark$ \\
\hline & Eccentricity & 1 & $\checkmark$ & $\checkmark$ \\
\hline \multirow{2}{*}{ Position } & (x,y) - min, mean, max & 6 & $\checkmark$ & $\checkmark$ \\
\hline & superpixel mask (8x8) & 64 & $\checkmark$ & $\checkmark$ \\
\hline \multirow{2}{*}{ Color } & rgb+lab (mean, std) & 6 & $\checkmark$ & $\checkmark$ \\
\hline & rgb+lab (histogram) & 48 & $\checkmark$ & $\checkmark$ \\
\hline High-dim & SIFT BoW & 400 & $\checkmark$ & \\
\hline \multirow{3}{*}{ Contextual } & contextual rgb+lab (mean, std) & 6 & $\checkmark$ & \\
\hline & contextual rgb+lab (histogram) & 48 & $\checkmark$ & \\
\hline & contextual SIFT BoW & 400 & $\checkmark$ & \\
\hline
\end{tabular}

\begin{tabular}{|c|c|c|c|c|}
\multicolumn{7}{c|}{ (b) Point Cloud Features. } \\
\hline Type & Name & Dim & Low & High \\
\hline \multirow{3}{*}{ Size } & Length proxy $-\lambda_{1}$ & 1 & $\checkmark$ & $\checkmark$ \\
\cline { 2 - 5 } & Area proxy $-\sqrt{\lambda_{1} \lambda_{2}}$ & 1 & $\checkmark$ & $\checkmark$ \\
\cline { 2 - 5 } & Volume proxy $-\sqrt[3]{\lambda_{1} \lambda_{2} \lambda_{3}}$ & 1 & $\checkmark$ & $\checkmark$ \\
\hline \multirow{3}{*}{ Shape } & Scatter $-\lambda_{3} / \Lambda$ & 1 & $\checkmark$ & $\checkmark$ \\
\cline { 2 - 5 } & Planarity $-\left(\lambda_{2}-\lambda_{3}\right) / \Lambda$ & 1 & $\checkmark$ & $\checkmark$ \\
\hline & Linearity - $\left(\lambda_{1}-\lambda_{2}\right) / \Lambda$ & 1 & $\checkmark$ & $\checkmark$ \\
\hline Position & $z-z_{g n d}-\min , \operatorname{mean}, \max$ & 3 & $\checkmark$ & $\checkmark$ \\
\hline \multirow{2}{*}{ Orientation } & Verticalness $-v_{1 z}$ & 1 & $\checkmark$ & $\checkmark$ \\
\cline { 2 - 5 } & Horizontalness $-\sqrt{1-v_{1 z}^{2}}$ & 1 & $\checkmark$ & $\checkmark$ \\
\hline High-dim & Spin image BoW & 1000 & $\checkmark$ & \\
\hline
\end{tabular}

\section{Classification}

The next step of our algorithm is to classify each segment. Classification is learning a mapping from feature vector of length $N$ to a label $l \in\{1, \ldots, L\}$. A soft classifier learns a mapping from feature space $\mathbb{R}^{N}$ to a probability mass function (pmf) vector over $L$ labels $\Delta^{L}$, where $\Delta \in[0,1]$.
We have empirically found the Random Forest (RF) classifier to be effective and use the implementation from [28].

A well-known challenge in semantic segmentation problems is class imbalance [7] [9]. In semantic segmentation problems, stuff classes tend to dominate the majority of the datapoints. Without addressing the class imbalance issue, a classifier typically learns to disregard the rare classes, resulting in zero or near-zero recall of those classes. This is addressed in [7] and [9] by subsampling their large training dataset at different rates based on object class occurrence, artificially boosting the representation of rare classes during classifier training. We take a similar approach, but address the issue by reweighting our samples rather than subsampling, due to a more limited training set size. Specifically, we calculate the probability distribution over label classes in the training set, mix it evenly with a uniform distribution, and artificially reweight our training examples to reflect this new mixed distribution. This procedure is performed when training all of our classifiers. The performance of the unimodal classifiers is explored in Section III-C.

\section{Fusion}

There are two choices for fusion: early and late. With two sensing modalities, three classifiers are trained in both cases: an image classifier, a point cloud classifier, and a fusion classifier, which operates on regions with overlapping sensor coverage. Let feature dimensions be $N_{i m g}$ and $N_{p c}$ in the image and point cloud domain, respectively.

In early fusion, the overlapping region between the two sensing modalities is first computed. The two unimodal classifiers, $P_{i m g}$ and $P_{p c}$, are then evaluated only on regions which are only covered by a single sensor.

$$
P_{i m g}: \mathbb{R}^{N_{i m g}} \rightarrow \Delta^{L}, P_{p c}: \mathbb{R}^{N_{p c}} \rightarrow \Delta^{L}
$$

In regions with overlapping coverage, a fusion classifier is trained and evaluated on a separate feature set of length $N_{p c+i m g}$, e.g. the concatenation of the unimodal features.

$$
P_{\text {earlyfusion }}: \mathbb{R}^{N_{p c+i m g}} \rightarrow \Delta^{L}
$$

In a late fusion architecture, classifiers are first run on each sensing modality separately, as described in Equation 1. A second level of classification is performed to fuse the results. We use the concatenation of the output pmf vectors from the image and point cloud modality classifiers, and learn the following mapping.

$$
P_{\text {latefusion }}: \Delta^{2 L} \rightarrow \Delta^{L}
$$

We run the fusion algorithm on extremely fine superpixels, as shown in Figure 3(b), to protect against label straddling. We take the stacking approach proposed in [18] to generate training data for the fusion classifier. The original training data is split into $k=2$ folds. Unimodal classifiers are trained on each set of $k-1$ folds and evaluated on the remaining fold. Those results are then used to train the fusion classifier.

An advantage of early fusion is that the joint feature space between the modalities is potentially more expressive. 
The early fusion strategy proposed in [3] uses simple lowdimensional feature vectors. By contrast, we opt to use higher-dimensional expressive features. As such, the learning problem becomes more difficult, as the classifier must learn a mapping from a high dimensional feature space $\mathbb{R}^{N_{p c+i m g}}$. Furthermore, since fusion only applies to overlapping regions, it does not enjoy the abundance of training data available to unimodal classifiers. In the case of KITTI data, as shown in Figure 2, the point cloud covers approximately $60 \%$ of the field-of-view (FOV) of the image, and the frontward facing camera only covers approximately $25 \%$ of the $360^{\circ} \mathrm{FOV}$ of the point cloud.

In the late fusion architecture, the inputs to the fusion classifier are the outputs of the unimodal classifiers. The unimodal classifiers are trained on the entire training set in each modality, not merely the overlapping region, thus enjoying an abundance of training data. The pmfs serve as a compact and expressive mid-level feature. The performance gain from late fusion is explored in Section III-D.

\section{E. Post-processing Graphical Model}

The CRF framework is commonly used for balancing local beliefs while enforcing spatial consistency [8] [9] [10] [11] [12] [13] [14]. Let data D represent an image and a registered point cloud from a single acquisition, $\left\{X_{i}\right\}$ be the set of superpixels from the image, $\left|X_{i}\right|_{P}$ be the perimeter of superpixel $i,\left|X_{i j}\right|_{P}$ be the length of the boundary between superpixels $i$ and $j$, and $d_{i}$ be the depth of superpixel $i$. We wish to infer label $z_{i} \in\{1, \ldots, L\}$ of superpixel $i$. We denote the classification scheme as detailed so far, where segments are classified using a unimodal classifier, and overlapping regions are passed through a late fusion classifier, by $P_{\text {unary }}\left(x_{i} \mid \mathbf{D}\right)$, where $x_{i} \in \mathbb{R}^{L}$ is the output pmf vector of superpixel $i$. The unary potential in the CRF framework is defined to the following:

$$
\Psi_{i}\left(z_{i}=l \mid x_{i}, \mathbf{D}, \mathbf{w}\right)=w_{\text {unary }}\left|X_{i}\right|_{P} P_{\text {unary }}\left(x_{i}^{l} \mid \mathbf{D}\right)
$$

The unary potential is the classified pmf, weighted by the superpixel perimeter. The weighting prevents small superpixels from overly influencing large neighboring superpixels.

A simple associative pairwise term is used to enforce spatial consistency, as follows:

$$
\Psi_{i j}\left(z_{i}=l, z_{j}=m \mid \mathbf{w}\right)=w_{\text {pair }}\left|X_{i j}\right|_{P} \delta(l, m) e^{\frac{-\left(d_{i}-d_{j}\right)^{2}}{2 \sigma_{d}^{2}}}
$$

We choose the strength of the pairwise term to be proportional to the length of the shared boundary, as superpixels with a large shared boundary are more likely to belong to the same object class. The strength of the pairwise is term is also weighted by a function of the difference in the median depths of the superpixels, which are estimated by projecting the 3D point clouds onto the image.

Summing over the unary and pairwise terms gives an energy, as shown in Equation 6, which describes the likelihood of a labeling over all superpixels in the whole image. Set $V$ describes the set of all superpixels, and set $E$ describes the

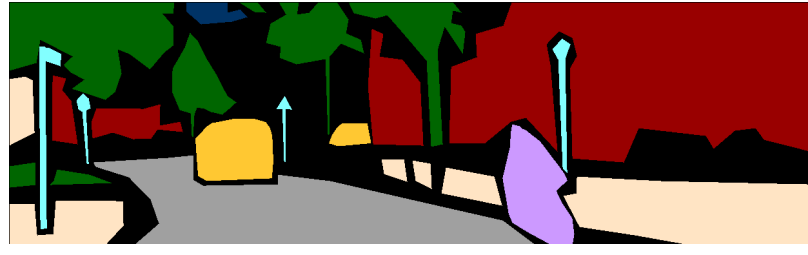

(a)

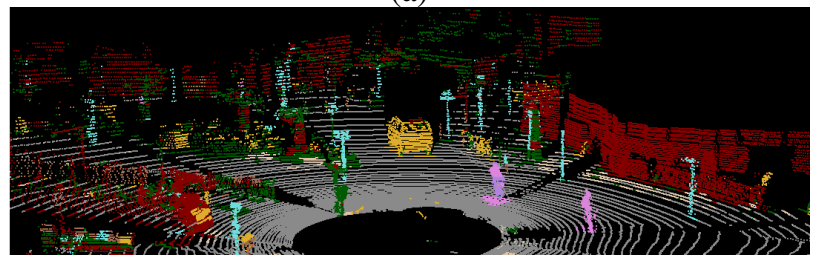

(b)

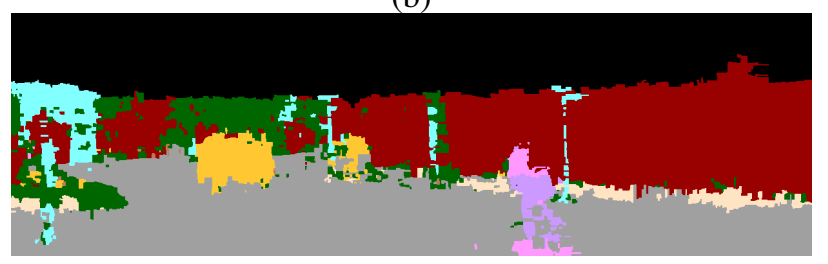

(c)

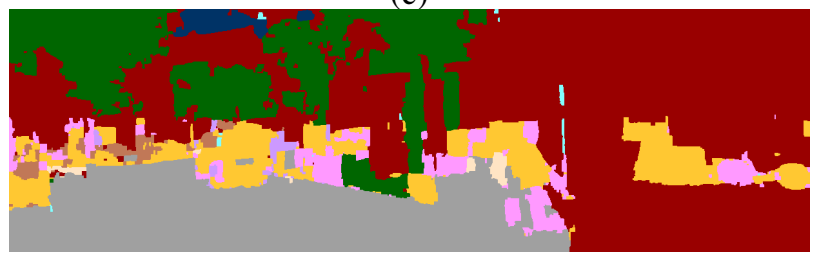

(d)

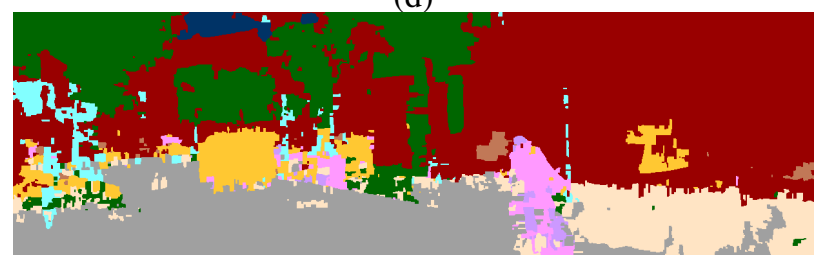

(e)

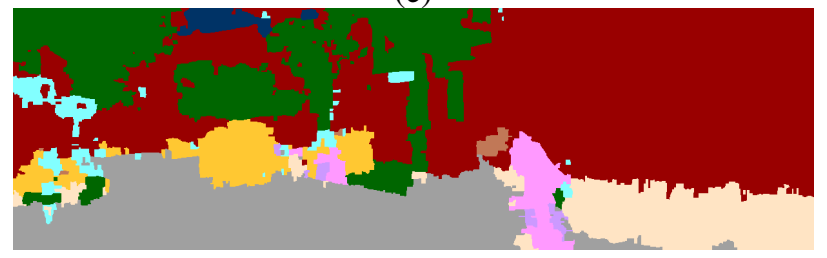

(f)

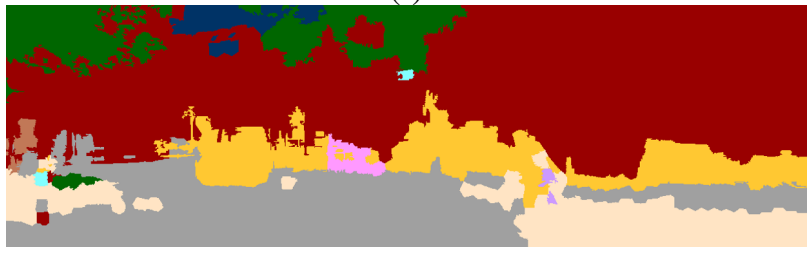

(g)

Fig. 5: (a) Ground truth; (b) point cloud unimodal classification; (c) point cloud unimodal semantic segmentation projected onto image; (d) image unimodal semantic segmentation; (e) fused semantic segmentation; (f) our overall

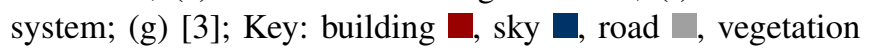
$\square$, sidewalk , car $\square$, pedestrian $\square$, cyclist $\square$, sign/pole $\square$, fence $\square$. 
set of all neighboring superpixels.

$$
E(\mathbf{z}, \mathbf{x}, \mathbf{D}, \mathbf{w})=-\sum_{i \in V} \Psi_{i}\left(z_{i} \mid x_{i}, \mathbf{D}, \mathbf{w}\right)-\sum_{(i, j) \in E} \Psi_{i j}\left(z_{i}, z_{j} \mid \mathbf{w}\right)
$$

The likelihood can be equivalently described as a probability distribution, as shown in Equation 7.

$$
P(\mathbf{z} \mid \mathbf{x}, \mathbf{D}, \mathbf{w})=\frac{1}{Z} \exp (-E(\mathbf{z}, \mathbf{x}, \mathbf{D}, \mathbf{w}))
$$

The most likely labeling is obtained by minimizing the energy, or equivalently, maximizing the probability.

$$
\mathbf{z}^{*}=\arg \min _{\mathbf{z}} E(\mathbf{z}, \mathbf{x}, \mathbf{D}, \mathbf{w})=\arg \max _{\mathbf{z}} P(\mathbf{z} \mid \mathbf{x}, \mathbf{D}, \mathbf{w})
$$

Since the pairwise terms are submodular, this can be efficiently and approximately solved using the well-known $\alpha$ expansion algorithm [29], which is a move-making graph cut algorithm.

\section{RESULTS}

\section{A. Dataset}

We have used the publicly available KITTI dataset [1] [2], consisting of image and 3D point clouds. Previously published results on semantic segmentation of the KITTI dataset [21] [3] used a limited ground truthed dataset, comprised of 45 and 25 acquisitions on the training and test sets, respectively. Furthermore, all acquisitions on the test set were from a single sequence. We annotated a set of 252 acquisitions from 8 sequences, and split the dataset into 140 and 112 acquisitions for training and testing, respectively, with 4 sequences in each. ${ }^{1}$ The split was chosen to balance object class representation in the sets. The labeled object classes were building, sky, road, vegetation, sidewalk, car, pedestrian, cyclist, sign/pole and fence. Performance was also evaluated over a coarser label set of building, sky, ground, vegetation and object, as used by Cadena and Košecká [3]. The ground class in the coarse label set consists of the road and sidewalk classes in the fine label set. The object class in the coarse label set is comprised of the car, pedestrian, cyclist, sign/pole, and fence classes.

\section{B. Comparison to state-of-the-art}

Results on the coarse and fine label sets on the images are shown in Tables II and III, respectively. The $1^{\text {st }}$ rows show performance of current state-of-the-art [3]. ${ }^{2}$ The last rows show the results of our system. ${ }^{3}$ On the coarse label set, as seen in Table II, the pixel-wise and classaverage performance of our system is $93.4 \%$ and $89.8 \%$, versus previous state-of-the-art [3] performance of $89.8 \%$ and $86.1 \%$, respectively. This represents a $35.3 \%$ reduction in incorrectly labeled pixels. Of the stuff categories, building

\footnotetext{
${ }^{1}$ Images were annotated using ground truthing tool [30]. Point clouds were annotated using an extension of CloudCompare [31] built in-house.

${ }^{2}$ Results were obtained by separately retraining and testing on each label set using publicly available code and verification from authors.

${ }^{3}$ Results for fine label set were obtained by training and testing on the fine label set. Results for the coarse label set were obtained by mapping the fine label set results onto the coarse label set.
}

and vegetation classes see significant increases, from $90.2 \%$ and $86.3 \%$ to $95.0 \%$ and $92.8 \%$, respectively, whereas $s k y$ performance decreases from $95.6 \%$ to $92.6 \%$. The most dramatic change is the object class, which covers all thing classes, which increases from $61.0 \%$ to $70.1 \%$.

On the fine label set, as seen in Table III, the pixelwise performance increase of our overall system is $89.3 \%$, compared to $84.1 \%$ from [3], a reduction of $32.7 \%$ of incorrectly labeled pixels. A more dramatic increase is seen in the class-average accuracy, from $52.4 \%$ to $65.4 \%$. Performance increases are seen on the stuff categories, but more dramatic increases are shown in the rare categories. For example, performance on the pedestrian class is more than doubled, from $28.6 \%$ to $65.1 \%$. Recall performance on the difficult cyclist, sign/pole, and fence classes are dramatically increased from $4.0 \%, 2.5 \%$, and $2.3 \%$ to $7.3 \%, 13.8 \%$, and $43.2 \%$, respectively.

The confusion matrix of our overall system is shown in Figure 6(d). High accuracy is achieved for stuff classes building, sky, road, and vegetation, all above $92.5 \%$. The sidewalk class is difficult to distinguish from road, but our algorithm is able to take advantage of cues from multiple modalities to achieve high performance of $69.7 \%$. The cyclist class only achieves $7 \%$ recall, but is often confused with the similarly looking pedestrian class $73 \%$ of the time. The fence class performs adequately at $43 \%$, and is often confused with vegetation, due to its proximity in the test set, and sometimes with building, due the similarity in shape and appearance. The sign/pole class remains difficult, but thing classes car and pedestrian perform well.

The $2^{\text {nd }}, 3^{\text {rd }}$, and $4^{\text {th }}$ rows of Tables II and III show the performance of our system at various stages: after persuperpixel image classifiers, after late-fusion, and finally, after the post-processing $\mathrm{CRF}$, and are discussed in further detail in Sections III-C, III-D, and III-E, respectively.

\section{Effect of multiple scales}

Table IV shows a performance comparison of the unimodal classifiers in the image and point cloud domains, given information at various scales: low-level only, high-level only, and low \& high-level features together. In both domains, using low-level information results in higher performance than high-level only, due to increased likelihood of oversegmentation at the high-level. Performance increases in both domains are observed when multiple scales are used. This is especially evident in the point cloud domain, which shows a $2.9 \%$ increase in pixel-wise accuracy and a dramatic $9.8 \%$ increase in class-average accuracy. Performance for every class except road is increased when using information from multiple scales. In particular, the recall performance for the cyclist class increases from $0 \%$ when using low or highlevel features only to $19.3 \%$ when using both scales. The building, car, pedestrian, sign/pole, and fence classes also see significant improvement.

An example segmentation using our image only classifier is shown in Figure 5(d), where ground truth is marked by Figure 5(a). In the example image, stuff classes such 


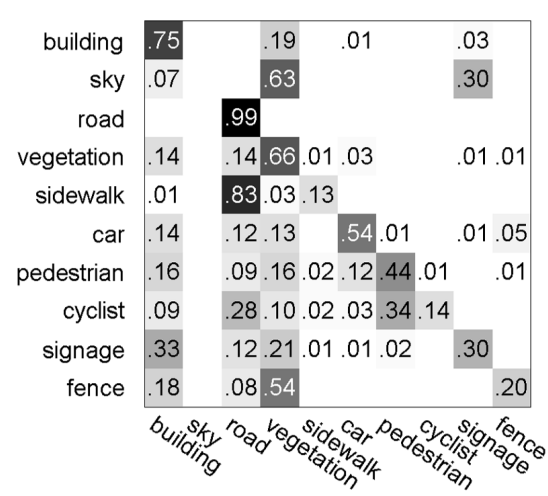

(a)

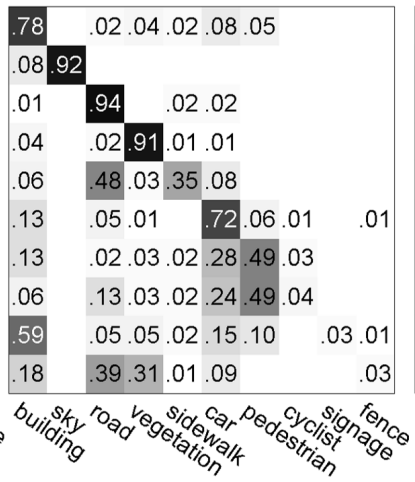

(b)

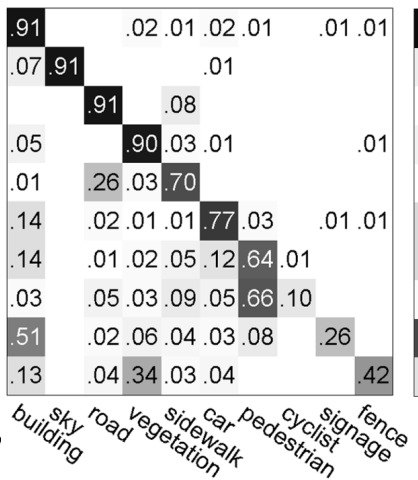

(c)

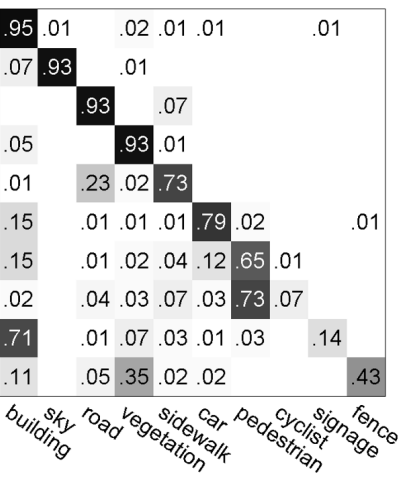

(d)

Fig. 6: Confusion matrices on overlapping sensor region; (a) Point cloud unimodal classifier; (b) Image unimodal classifier; (c) Late-fused classifier; (d) Confusion matrix of our overall system on image region.

TABLE II: Coarse label set performance comparison against state-of-the-art; glob: pixel-wise accuracy, class: class-average accuracy, bldg: building, sky: sky, gnd: ground, veg: vegetation, obj: object.

\begin{tabular}{|c|c|c|c|c|c|c|c|c|c|c|}
\hline & \multicolumn{7}{|c|}{ Performance } & \multicolumn{3}{|c|}{ Information Used } \\
\hline & glob & class & bldg & sky & gnd & veg & obj & img & $\mathrm{pc}$ & crf \\
\hline Cadena and Košecká [3] & $89.8 \%$ & $86.1 \%$ & $90.2 \%$ & $95.6 \%$ & $97.4 \%$ & $86.3 \%$ & $61.0 \%$ & $\bar{\checkmark}$ & $\sqrt{ }$ & $\checkmark$ \\
\hline Ours (image only) & $89.4 \%$ & $86.1 \%$ & $87.5 \%$ & $92.5 \%$ & $91.9 \%$ & $92.5 \%$ & $66.1 \%$ & $\checkmark$ & & \\
\hline Ours (late fused) & $92.8 \%$ & $89.3 \%$ & $93.5 \%$ & $92.5 \%$ & $98.1 \%$ & $92.0 \%$ & $70.4 \%$ & $\checkmark$ & $\checkmark$ & \\
\hline Ours (CRF) & $93.4 \%$ & $89.8 \%$ & $95.0 \%$ & $92.6 \%$ & $98.5 \%$ & $92.8 \%$ & $70.1 \%$ & $\checkmark$ & $\checkmark$ & $\checkmark$ \\
\hline
\end{tabular}

TABLE III: Fine label set performance comparison against state-of-the-art; glob: pixel-wise accuracy, class: class-average accuracy, bldg: building, sky: sky, road: road, veg: vegetation, side: sidewalk, car: car, ped: pedestrian, cyc: cyclist, sgn: sign/pole, fnc: fence.

\begin{tabular}{|c|c|c|c|c|c|c|c|c|c|c|c|c|}
\hline & glob & class & bldg & sky & road & veg & side & car & ped & cycl & $\operatorname{sgn}$ & fnc \\
\hline Cadena and Košecká [3] & $84.1 \%$ & $52.4 \%$ & $92.5 \%$ & $95.7 \%$ & $92.5 \%$ & $86.3 \%$ & $51.5 \%$ & $67.9 \%$ & $28.6 \%$ & $4.0 \%$ & $2.5 \%$ & $2.3 \%$ \\
\hline Ours (image only) & $83.5 \%$ & $53.3 \%$ & $87.5 \%$ & $92.5 \%$ & $94.5 \%$ & $92.5 \%$ & $34.5 \%$ & $71.4 \%$ & $49.0 \%$ & $3.6 \%$ & $4.1 \%$ & $3.3 \%$ \\
\hline Ours (late fused) & $88.0 \%$ & $64.8 \%$ & $93.5 \%$ & $92.5 \%$ & $91.2 \%$ & $92.0 \%$ & $69.7 \%$ & $76.5 \%$ & $63.7 \%$ & $10.0 \%$ & $16.6 \%$ & $42.2 \%$ \\
\hline Ours (CRF) & $89.3 \%$ & $65.4 \%$ & $95.0 \%$ & $92.6 \%$ & $92.6 \%$ & $92.8 \%$ & $73.3 \%$ & $78.7 \%$ & $65.1 \%$ & $7.3 \%$ & $13.8 \%$ & $43.2 \%$ \\
\hline
\end{tabular}

TABLE IV: Effect of incorporating multiple scales; glob: pixel-wise accuracy, class: class-average accuracy, bldg: building, sky: sky, road: road, veg: vegetation, side: sidewalk, car: car, ped: pedestrian, cyc: cyclist, sgn: sign/pole, fnc: fence.

\begin{tabular}{|c|c|c|c|c|c|c|c|c|c|c|c|c|c|c|}
\hline & \multicolumn{2}{|c|}{ Scale } & \multicolumn{12}{|c|}{ Performance } \\
\hline & Low & High & glob & class & bldg & sky & road & veg & side & car & ped & cycl & sgn & fnc \\
\hline \multirow{3}{*}{ img } & $\mathrm{X}$ & & $83.0 \%$ & $52.6 \%$ & $86.6 \%$ & $92.8 \%$ & $94.1 \%$ & $93.6 \%$ & $29.6 \%$ & $67.8 \%$ & $49.3 \%$ & $6.1 \%$ & $5.1 \%$ & $0.8 \%$ \\
\hline & & $\mathrm{x}$ & $81.1 \%$ & $50.4 \%$ & $88.7 \%$ & $93.5 \%$ & $90.1 \%$ & $90.6 \%$ & $20.6 \%$ & $71.4 \%$ & $42.0 \%$ & $3.1 \%$ & $3.8 \%$ & $0.1 \%$ \\
\hline & $\mathrm{X}$ & $\mathrm{x}$ & $83.5 \%$ & $53.3 \%$ & $87.4 \%$ & $92.5 \%$ & $94.5 \%$ & $92.5 \%$ & $34.5 \%$ & $71.4 \%$ & $49.0 \%$ & $3.6 \%$ & $4.1 \%$ & $3.3 \%$ \\
\hline \multirow{3}{*}{$\mathrm{pc}$} & $\mathrm{x}$ & & $67.1 \%$ & $38.6 \%$ & $72.9 \%$ & - & $89.4 \%$ & $53.9 \%$ & $26.1 \%$ & $36.0 \%$ & $22.4 \%$ & $0.0 \%$ & $30.4 \%$ & $16.5 \%$ \\
\hline & & $\mathrm{X}$ & $59.5 \%$ & $32.4 \%$ & $68.0 \%$ & - & $97.8 \%$ & $11.7 \%$ & $3.8 \%$ & $34.6 \%$ & $35.8 \%$ & $0.0 \%$ & $38.3 \%$ & $1.7 \%$ \\
\hline & $\mathrm{x}$ & $\mathrm{x}$ & $70.0 \%$ & $49.8 \%$ & $86.9 \%$ & - & $89.2 \%$ & $55.0 \%$ & $26.2 \%$ & $50.0 \%$ & $49.0 \%$ & $19.3 \%$ & $51.7 \%$ & $21.1 \%$ \\
\hline
\end{tabular}

as building, sky, vegetation, road are generally classified correctly. However, small objects are difficult to accurately label, as parts of the cyclist are misclassified as car, and the pole on the left of the image is completely missed. Pieces of the car are also misclassified, along with the sidewalk on the bottom right.

\section{Fusion results}

Figure 5(b) shows the point cloud classification for the example acquisition, and Figure 5(c) shows its projection onto the image. As seen in Figure 5(c), the point cloud unimodal classifier performs better at classifying the cyclist, which is labeled as cyclist and pedestrian. The pole on the left of the image and almost the entire car are classified correctly as well. Significant qualitative improvement is seen after fusion, as shown in Figure 5(e). Specifically, the cyclist is classified as the easily confusable pedestrian class, and the pole and car are correctly classified. Even the sidewalk on the bottom right side is correctly identified, despite being classified as road in the point cloud domain and building in the image domain.

Quantitatively, our system receives a strong boost after sensor fusion. In the fine label set, as shown in Table III, pixel-wise performance is increased from $83.5 \%$ to $88.0 \%$, and class-average performance is increased from $53.3 \%$ to $64.8 \%$, and performance increases are seen in almost every object class. At this stage, our system outperforms the current state-of-the-art [3] in both label sets, as shown in the $1^{\text {st }}$ and 
$3^{\text {rd }}$ rows of Tables II and III.

Figures 6(a) and 6(b) show the performance of the 3D point cloud and image unimodal classifiers, respectively, on the overlapping region of the two modalities. Figure 6(c) shows the confusion matrix on the overlapping region after fusion. A few interesting cases where fusion greatly improves performance are discussed below.

The sidewalk class is difficult to distinguish from road. In the point cloud, they have near identical shape appearance, with the only distinguishing feature being a slight elevation difference. In the image domain, sidewalk is more accurately classified due to color and horizontal position features, but is still misclassified as road the majority of the time. After fusion, however, sidewalk is classified correctly $70 \%$ of the time, a dramatic increase from $13 \%$ and $35 \%$ in the individual domains, as seen in Figures 6(a)(b)(c).

The fusion classifier is also able to learn modes of failure. For example, recall on the fence class is $42 \%$ after fusion, higher than the combined recall of $20 \%$ and $3 \%$ in individual domains, as seen in Figure 6. The fusion classifier learns that a segment classified as road in the image and building or vegetation in the point cloud may in actuality be fence.

\section{E. Post-proessing CRF}

The parameters for the CRF was set to $w_{\text {unary }}=10$, $w_{\text {pair }}=5.63$ using cross validation and a parameter sweep. Figure 5(f) shows a final semantic segmentation after the post-processing CRF. Compared to Figure 5(e), there is a qualitative improvement, as the result is much more spatially consistent. Quantitatively, as seen in the last two rows of Tables II and III, slight improvements are made in both pixelwise and class-average accuracy. For example, on the fine label set, global and class-average accuracies are increased from $88.0 \%$ to $89.3 \%$ and $64.8 \%$ to $65.4 \%$, respectively.

\section{CONCLUSIONS}

We proposed a semantic segmentation algorithm which fuses information from multiple modalities. The algorithm effectively integrated information at multiple scales by performing multiple levels of segmentation and augmenting feature vectors on low-level segments with their associated high-level segments. A late fusion architecture was used to effectively leverage information from single modalities. Finally, a CRF was used as a post-processing step to balance local beliefs while enforcing spatial consistency. The algorithm was validated on image and point cloud data from the KITTI platform, and shown to achieve state-of-the-art results on the problem of urban semantic segmentation.

A few challenges continue to remain. The cyclist class is often confused for pedestrian, and sign/pole remains difficult to identify. Integrating top-down detectors for thing classes in both modalities would be a future direction of research. Another future direction is integrating temporal information from per-acquisition imagery and point cloud scans. Integrating our results with reconstruction algorithms would be an interesting research direction as well.

\section{ACKNOWLEDGMENTS}

This work was supported by the U.S. Department of Homeland Security under Grant Award 2011-DN-077-ARI049-03.

\section{REFERENCES}

[1] A. Geiger, P. Lenz, C. Stiller, and R. Urtasun, "Vision meets robotics: The kitti dataset," 2013.

[2] A. Geiger, P. Lenz, and R. Urtasun, "Are we ready for autonomous driving? the kitti vision benchmark suite," in CVPR, 2012.

[3] C. Cadena and J. Košecká, "Semantic segmentation with heterogeneous sensor coverages," in ICRA, 2014.

[4] C. Farabet, C. Couprie, L. Najman, and Y. LeCun, "Scene parsing with multiscale feature learning, purity trees, and optimal covers," ICML, 2012.

[5] R. Girshick, J. Donahue, T. Darrell, and J. Malik, "Rich feature hierarchies for accurate object detection and semantic segmentation," in $C V P R, 2014$

[6] J. Tighe and S. Lazebnik, "Superparsing: Scalable nonparametric image parsing with superpixels," in ECCV, 2010.

[7] — , "Finding things: Image parsing with regions and per-exemplar detectors," in CVPR, 2013.

[8] G. Singh and J. Kosecka, "Nonparametric scene parsing with adaptive feature relevance and semantic context," in CVPR, 2013.

[9] J. Yang, B. Price, S. Cohen, and M.-H. Yang, "Context driven scene parsing with attention to rare classes," CVPR, 2014.

[10] D. Anguelov, B. Taskarf, V. Chatalbashev, D. Koller, D. Gupta, G. Heitz, and A. Ng, "Discriminative learning of markov random fields for segmentation of $3 \mathrm{~d}$ scan data," in CVPR, 2005.

[11] D. Munoz, N. Vandapel, and M. Hebert, "Directional associative markov network for 3-d point cloud classification," in 3DPVT, 2008.

[12] D. Munoz, J. A. Bagnell, N. Vandapel, and M. Hebert, "Contextual classification with functional max-margin markov networks," in CVPR, 2009.

[13] R. Shapovalov and A. Velizhev, "Cutting-plane training of nonassociative markov network for $3 \mathrm{~d}$ point cloud segmentation," in 3DIMPVT, 2011.

[14] Y. Lu and C. Rasmussen, "Simplified markov random fields for efficient semantic labeling of 3d point clouds," in IROS, 2012.

[15] P. Kohli, P. H. Torr et al., "Robust higher order potentials for enforcing label consistency," IJCV, 2009.

[16] L. Ladickỳ, P. Sturgess, K. Alahari, C. Russell, and P. H. Torr, "What, where and how many? combining object detectors and crfs," in ECCV, 2010.

[17] L. Ladicky, C. Russell, P. Kohli, and P. H. Torr, "Associative hierarchical crfs for object class image segmentation," in ICCV, 2009.

[18] D. Munoz, J. A. Bagnell, and M. Hebert, "Stacked hierarchical labeling," in $E C C V, 2010$.

[19] X. Xiong, D. Munoz, J. A. Bagnell, and M. Hebert, "3-d scene analysis via sequenced predictions over points and regions," in ICRA, 2011.

[20] D. Munoz, J. A. Bagnell, and M. Hebert, "Co-inference machines for multi-modal scene analysis," in ECCV, 2012.

[21] S. Sengupta, E. Greveson, A. Shahrokni, and P. H. Torr, "Urban 3d semantic modelling using stereo vision," in ICRA, 2013.

[22] H. He and B. Upcroft, "Nonparametric semantic segmentation for $3 \mathrm{~d}$ street scenes," in IROS, 2013.

[23] P. Arbeláez, J. Pont-Tuset, J. Barron, F. Marques, and J. Malik, "Multiscale combinatorial grouping," in CVPR, 2014.

[24] J. Papon, A. Abramov, M. Schoeler, and F. Worgotter, "Voxel cloud connectivity segmentation supervoxels for point clouds," in CVPR, 2013.

[25] M. Himmelsbach, T. Luettel, and H. Wuensche, "Real-time object classification in $3 \mathrm{~d}$ point clouds using point feature histograms," in IROS, 2009.

[26] D. G. Lowe, "Distinctive image features from scale-invariant keypoints," in IJCV, 2004.

[27] A. E. Johnson and M. Hebert, "Using spin images for efficient object recognition in cluttered 3d scenes," in PAMI, 1999.

[28] P. Dollár, "Piotr's Image and Video Matlab Toolbox (PMT)," http://vision.ucsd.edu/ pdollar/toolbox/doc/index.html.

[29] Y. Boykov, O. Veksler, and R. Zabih, "Fast approximate energy minimization via graph cuts," PAMI, 2001.

[30] D. Hoiem, "Software," http://web.engr.illinois.edu/ dhoiem/, accessed: 2014-09-14

[31] "Cloudcompare," http://www.danielgm.net/cc/, accessed: 2014-09-14. 